\title{
Small-scale Substructure in Dark Matter Haloes: Where Does Galaxy Formation Come to an End?
}

\author{
J. E. Taylor, J. Silk \\ Astrophysics, University of Oxford \\ Denys Wilkinson Building, Keble Road, Oxford, OX1 3RH, UK
}

\author{
A. Babul \\ Physics and Astronomy, University of Victoria \\ Elliott Building 3800 Finnerty Road, Victoria, BC, V8P 1A1, Canada
}

\begin{abstract}
Models of structure formation based on cold dark matter predict that most of the small dark matter haloes that first formed at high redshift would have merged into larger systems by the present epoch. Substructure in present-day haloes preserves the remains of these ancient systems, providing the only direct information we may ever have about the low-mass end of the power spectrum. We describe some recent attempts to model halo substructure down to very small masses, using a semi-analytic model of halo formation. We make a preliminary comparison between the model predictions, observations of substructure in lensed systems, and the properties of local satellite galaxies.
\end{abstract}

\section{Introduction}

The goal of this panel session was to assess the problems faced by cold dark matter (CDM). We will discuss one of these in particular, the apparent conflict between theory and observations on sub-galactic scales, known variously as the substructure problem or the dwarf galaxy problem. In the standard CDM picture of galaxy formation, dark matter haloes are the sites for gas cooling and star formation, so there should be a close correspondence between luminous galaxies and individual dark matter haloes or 'subhaloes', the bound remnants of haloes that have merged into larger virialised systems. Theory and simulations predict that by $z=0$ the halo and subhalo mass functions should be relatively steep power laws on mass scales smaller than galaxy clusters. Galaxies, on the other hand, appear to have a limited range of luminosities and masses. The galaxy luminosity function can only be sampled down to its faintest limit in the very nearby universe, but it generally appears to be flat or slowly rising. Assuming a monotonic relation between average galaxy luminosity and halo mass, this leaves two possibilities; either CDM models are incorrect and there is little small-scale structure in the universe, or the net efficiency of galaxy formation (in the broadest sense of the amount of stellar light produced within an individual halo for a given halo mass) must decrease dramatically on small scales. 
There is also increasing observational evidence that the faint-end slope of the galaxy luminosity function varies systematically with environment (e.g. the talk by Tully and the poster by Roberts in these proceedings). Whatever mechanism suppresses the formation of dwarf galaxies, it must also reproduce this environmental dependence. Of course the dependence may not be primordial; effects such as galaxy harassment and ram-pressure stripping could have modified the luminosity function differently in different environments. The trend is the reverse of what one would naively expect, however - there are more dwarf galaxies per giant galaxy in high-density environments, where harassment and stripping might be expected to reduce their relative number.

On large scales there is now strong observational support for a cosmological model whose matter density is dominated by a dark, non-baryonic component (Spergel, this meeting). It would be possible to reproduce most of these observations with variants of CDM in which structure is reduced on small scales, such as warm, annihilating or collisional dark matter, or CDM with a truncated power spectrum; thus resolving the substructure problem. There are tentative indications from recent lensing observations that dark substructure has now been detected, however, so modifying CDM may be the wrong solution to the problem. In what follows we will assume the CDM picture of structure formation is correct on all scales, and discuss what the small-scale distribution of dark matter can then tell us about galaxy formation.

\section{A Brief History of the Dwarf Galaxy Problem}

The discrepancy between the field luminosity function and the predictions of hierarchical models was noted before the modern conception of CDM itself (White and Rees 1978). Kauffmann, White and Guiderdoni (1993) made the more specific observation that satellite galaxies should far outnumber the dozen dwarf companions seen around each giant galaxy in the Local Group. They proposed several possible solutions to the problem, but could not conclude definitively on the role of dynamics in disrupting dwarfs. It took high-resolution numerical simulations (Klypin et al. 1999; Moore et al. 1999) to prove convincingly that large numbers of subhaloes would survive in a system like the Milky Way.

In their analysis, Moore et al. (1999) assumed that the observed dwarfs trace out the full depth of their surrounding dark matter potential, and thus that the velocity dispersion of their stellar component is roughly $1 / \sqrt{2}$ times the peak circular velocity of the corresponding dark matter structure. This led to an overall discrepancy of a factor of 50 between the number of dwarfs and the number of subhaloes that could host them. Subsequently, White (2000) pointed out that the stars in observed dwarfs might be condensed systems within much larger potential wells. Detailed modeling (Stoehr et al. 2002; Hayashi et al. 2003) confirmed that the satellites of the Milky Way could in fact reside in the most massive subsystems within its halo without this being apparent from their velocity dispersion profiles.

These two different pictures of the suppression of galaxy formation on small scales are illustrated schematically in Fig. 1. In the first case (left-hand panel), visible satellites are rare objects (only one subhalo in 100 on the smallest scales), but the masses inferred from rotation curves (lower line) are close to the total 

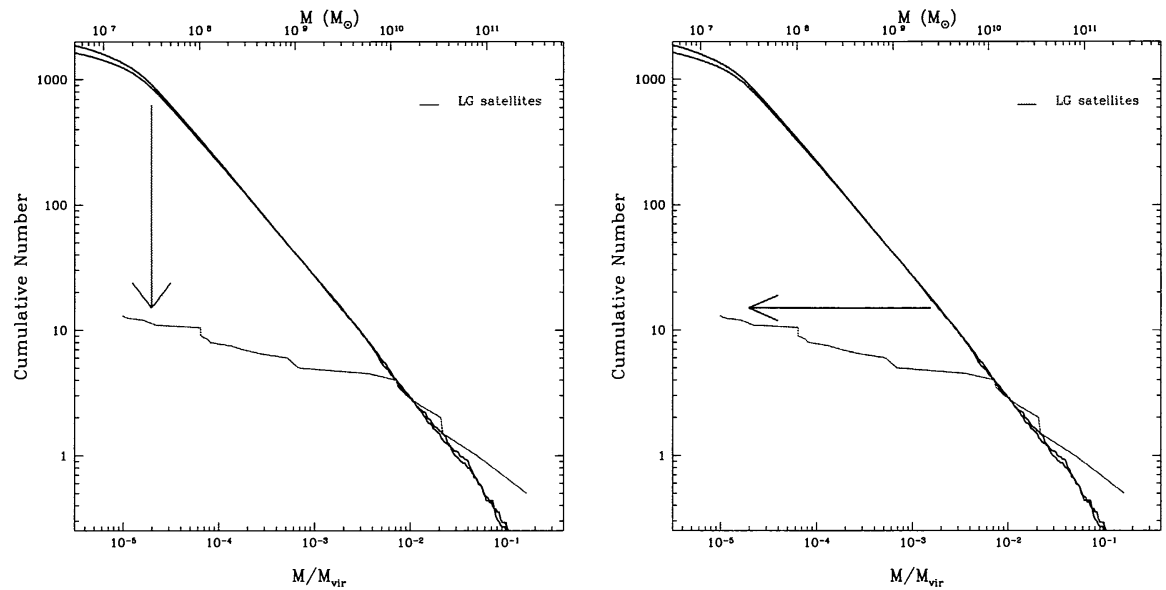

Figure 1. Two models for the suppression of dwarf galaxies. Model 1 (left-hand panel): visible dwarfs are rare but form over a wide range of halo mass; Model 2 (right-hand panel): dwarf galaxies form exclusively in the most massive haloes.

mass for each system (uppermost curve). In the second case (right-hand panel), dwarfs form with widely varying efficiency in the dozen most massive subsystems, and galaxy formation stops altogether below some fairly high mass cutoff. These two scenarios imply very different underlying regulatory mechanisms; a simple one depending only on the mass or circular velocity of the halo (e.g. Dekel \& Silk 1986; Stoehr et al. 2002) in the case of the latter model, or one based on some other property of the system such as its age (Bullock, Kravtsov, \& Weinberg 2000; Benson et al. 2002) in the case of the former. Frustratingly, even very detailed observations of dwarf luminosities and kinematics may not be able to distinguish between these possibilities (Stoehr et al. 2002). Additional information is needed.

\section{Modeling Substructure: Do We Know Overmerging is Over?}

Given the complex non-linear dynamics involved in structure formation, selfconsistent numerical simulations are the preferred method for studying CDM halo formation and halo substructure. Simulations suffer from one serious drawback, however - their results are least reliable in the densest parts of the halo, where the dynamical timescale is shortest and artificial heating has the greatest effect. The artificial smoothing of the density distribution in these regions is referred to as 'overmerging' (for a review of the problem see Moore 2000).

In early simulations of the formation of galaxy clusters, overmerging erased substructure completely (e.g. White 1976). When simulations reached sufficient resolution to resolve roughly as many subsystems as there are galaxies in a cluster, the problem was considered 'solved' (e.g. Ghigna et al. 2000), although the scale invariance of halo properties quickly lead to an excess dwarf satellite problem in galaxy haloes. Interestingly enough, the assumption that the over- 


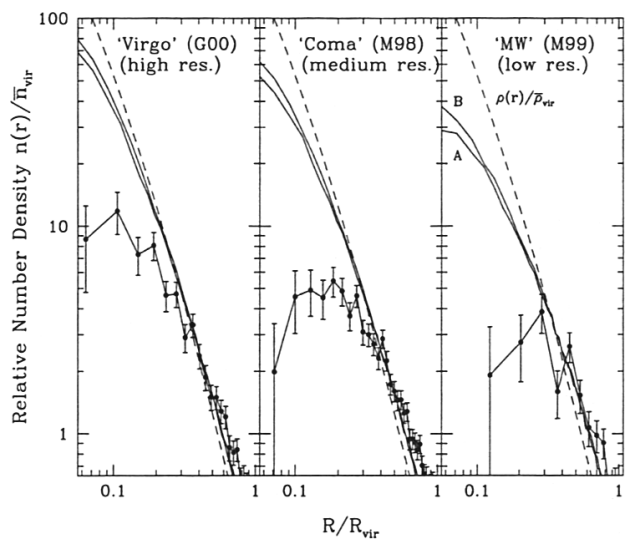

Figure 2. The number density of subhaloes in three simulations of increasing resolution from right to left (jagged lines with error bars - from Moore et al. 1998, 1999; Ghigna et al. 2000), compared with the semi-analytic prediction (smooth solid lines) and the halo density profile (dashed line). Each quantity is normalised to its mean value within the virial radius.

merging problem is now solved has not been fully tested. At current mass and force resolution, simulations converge on the properties of massive subhaloes in the outermost regions of a halo (e.g. Stoehr 2002). Convergence tests in the innermost regions, where overmerging should be strongest, have not been presented. These regions contain only a small fraction of the total substructure in any given mass range, so central overmerging would not be apparent in convergence tests averaged over the whole halo; for applications such as lensing, however, central substructure is crucially important.

As an independent test of overmerging, we have compared the substructure in several high-resolution simulations of CDM haloes with the subhalo populations predicted by a semi-analytic model of halo formation (Taylor \& Babul 2001, 2003; Taylor 2001). Fig. 2 compares the spatial distribution of subhaloes in the semi-analytic (upper solid lines) and numerical (lower lines with error bars). In the outer regions, the two techniques agree on the normalisation of the halo mass function. In the inner regions, the semi-analytic model, which should not be affected by overmerging, predicts up to ten times more subhaloes. It is not clear which of these results is correct - the disruption rate in the semi-analytic model could be artificially low. Increasing the resolution of the simulations (three panels, from right to left) increases the amount of central substructure, however, with no sign of convergence in the simulations presented here. This suggests that the simulations probably still underestimate the amount of central substructure in haloes, while the semi-analytic model may provide a more reliable estimate.

The existence of small-scale structure can be confirmed observationally by detecting its gravitational effects in lensing systems. Small structures in the halo of a lens can produce flux ratios between pairs or sets of images that cannot be explained by a smooth potential (Schneider, this meeting). The analysis of 

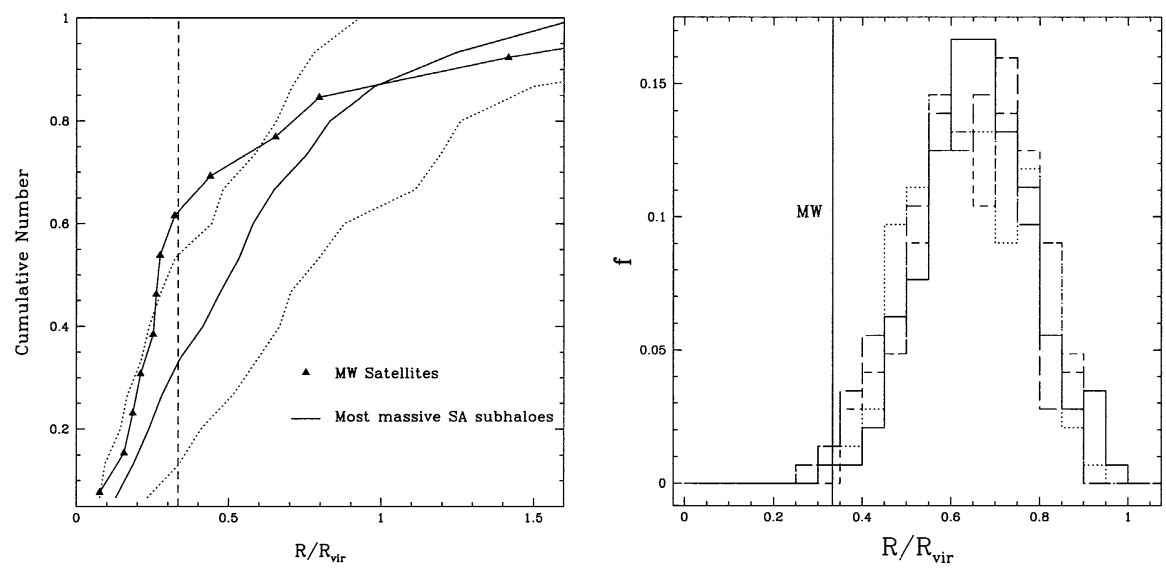

Figure 3. (Left-hand panel) The cumulative number of the dozen most massive subhaloes within some fraction of the virial radius. The solid line shows the average distribution for all 144 models, while the dotted lines show the $10 \%$ and $90 \%$ contours of the distribution. The triangles show the distribution of Milky Way satellites. (Right-hand panel) Histograms of the fraction of the virial radius containing 8 of the 11 most massive subhaloes, for each of the 144 model haloes. The different line styles are for different model parameters; the vertical line shows the value for the Milky Way.

several systems has already produced evidence for halo substructure amounting to $1-2 \%$ of the projected mass density of the inner parts of lensing systems (Dalal \& Kochanek 2002), but these estimates may be affected by stellar microlensing or other complicating effects. Future observations of the bending of background radio jets (Metcalf 2002) or spectrally resolved flux anomalies (Metcalf et al. 2003) will clarify the situation.

\section{Satellites vs. Subhaloes}

We will now discuss the average properties of substructure in a set of 144 semianalytic haloes, generated in a Lambda-CDM cosmology with parameters consistent with recent WMAP results (Spergel, this meeting). The haloes have a final mass of $1.6 \times 10^{12} M_{\odot}$ and their merger histories are resolved down to $5 \times 10^{7} M_{\odot}$ (given the typical mass loss of subhaloes, this means the final substructure mass function is mostly complete down to a few million solar masses) and back to $z=30$. We will consider whether dwarf galaxies can be associated with the subhaloes as a function of their original mass (before tidal stripping in larger systems), their original circular velocity, or their age.

\subsection{The Clustering of Satellites}

Fig. 3 shows the cumulative radial distribution of the dozen subhaloes with the greatest original masses, normalised to the present-day virial radius of the 

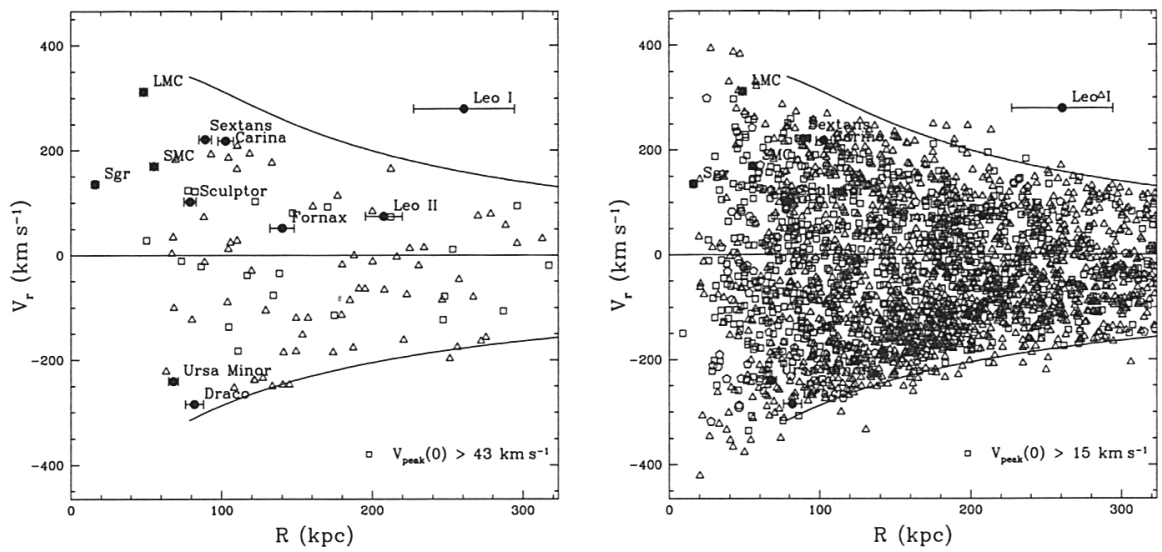

Figure 4. Positions and radial velocities of Milky Way satellites (solid circles with error bars), compared to the distribution of massive subhaloes (left-hand panel; open symbols) and low-mass subhaloes (righthand panel). The solid lines indicate the envelope of the distribution, and the symbol types indicate the merger epoch (see text).

halo, and averaged over the full set of trees. The dotted lines indicate the $10 \%$ and $90 \%$ contours of the distribution. The solid line and triangles show the distribution of satellites for the Milky Way, assuming a virial radius of $314 \mathrm{kpc}$, corresponding to a mass of $1.6 \times 10^{12} M_{\odot}$. If this normalisation is correct, then the satellites of the Milky Way are clearly more clustered than the most massive subhaloes in its halo should be.

To illustrate the statistical strength of this conclusion more clearly, Fig. 3 shows histograms of the normalised radius containing the innermost 8 of the 11 most massive satellites, for all 144 model haloes. The different line styles show results for different values for the Coulomb logarithm, the mass-loss parameter or the mean orbital circularity in the semi-analytic model (see Taylor \& Babul 2003). The vertical line indicates the value for the Milky Way. No more than 1-2 of the 144 trees (depending on the model assumed) are as clustered as the satellites of the Milky Way. Thus, the visible satellites of the Milky Way are more clustered than its most massive subhaloes at a $\sim 3-\sigma$ level.

\subsection{Satellite Kinematics}

There is at least one important complication in the preceding analysis. The halo of the Milky Way could be less massive than assumed, and therefore have a smaller virial radius and a less extended population of satellites. In this case, the normalisation of the radii used in Fig. 3 would be incorrect. Reducing the virial radius of the Milky Way by $20-30 \%$ would bring the observed distribution into perfect agreement with the predictions for massive subhaloes; this would imply a total halo mass of $\sim 8 \times 10^{11} M_{\odot}$, which is still within the range of estimates for the Milky Way (e.g. Klypin, Zhao, \& Somerville 2002).

This possibility can be tested by looking at satellite kinematics. The local flow around the halo of the Milky Way is quite quiet; its satellites have large 
radial velocities because they have been accelerated by its potential. If the mass of the halo is smaller, the satellites will have lower radial velocities. Fig. 4 shows radial velocity versus radius, for the luminous satellites of the Milky Way (large filled circles with error bars) and for all the subhaloes over some (original) circular velocity limit (open symbols), selected from each of a small set of semianalytic haloes. The symbol types indicate the merger epoch $z_{\mathrm{m}}$ of the subhalo; triangles for $z_{\mathrm{m}}<2$, squares for $z_{\mathrm{m}}=2-4$, pentagons for $z_{\mathrm{m}}=4-6$, hexagons for $z_{\mathrm{m}}=6-8$, and circles for $z_{\mathrm{m}}>8$. The thick lines give a rough envelope for the distribution of subhaloes.

The left-hand panel shows the results for massive systems. Several points are worth noting. First, the observed satellites are concentrated at smaller radii than the most massive subhaloes (left-hand plot), as discussed previously. On the other hand, the observed scatter in radial velocity is consistent with the model, which assumes a total halo mass of $1.6 \times 10^{12} M_{\odot}$. Reducing this by a factor of 2 would reduce the velocity dispersion by $20-30 \%$, which is incompatible with the observed velocity of several satellites, notably the LMC. In fact even assuming this mass for the halo, there is no object like Leo I among the most massive subhaloes. Leo I has such a large radial velocity away from the Milky Way that it must have passed through the potential of its halo on a very radial orbit. Even in this case, however, if it had been massive it would have lost a substantial amount of orbital energy through dynamical friction. Thus Leo I cannot be a massive system.

The right-hand panel shows the same comparison for the less massive satellites. Once again, the envelope matches the observed scatter in radial velocities, suggesting the assumed mass of the halo is roughly correct. Now there are a few systems like Leo I; as before, they have passed close to the centre of the halo and are heading out on very radial orbits, but now their velocities are higher as they have not experienced as much frictional drag. While the subhaloes are still located at larger radii than the luminous satellites on average, there is a strong trend in the distribution with merger epoch. Selecting systems which merged into the halo before a redshift of 4-6 (pentagons, hexagons or circles) produces roughly the same degree of clustering as observed for the luminous satellites.

\section{Summary}

The predictions of large amounts of small-scale structure and substructure in CDM cosmologies is perhaps startling, but it is not in and of itself a reason to reject CDM. Galaxy formation is unlikely to be a simple process, particularly on small scales where many regulatory mechanisms might limit its efficiency. Thus, if we fail to see large numbers of dwarf galaxies in the nearby universe, this may be telling us more about galaxy formation than it does about CDM. Assuming CDM substructure exists, its properties can provide important constraints on the process of dwarf galaxy formation.

Simulations and semi-analytic models of halo substructure agree on its properties in the outer parts of haloes; in the inner regions, simulations may still sutfer from overmerging, the artificial disruption of substructure due to numerical effects. Interestingly, the amount of central substructure seen in the semi-analytic haloes considered here is consistent with the amount of substructure inferred 
from strong lensing experiments. Thus the semi-analytic haloes may provide a more accurate picture of the spatial distribution of substructure around galaxies.

Comparing this distribution to the observed location of the Milky Way's luminous satellites, it is clear that observed satellites are more clustered than the most massive subhaloes should be, at a $\sim 3 \sigma$ level. Satellite radial velocities strengthen this conclusion further, partly by constraining the mass of the halo and partly through specific examples such as Leo I. This appears to rule out models of feedback where only the most massive subhaloes host dwarf galaxies, and favours models in which the oldest subhaloes do instead. More generally, it should also rule out many proposed alternatives to CDM, such as warm or interacting dark matter. Almost all of these scenarios would destroy the smallest subhaloes and those in the centre of the main system preferentially, which is the exact opposite of the pattern observed.

Acknowledgments. JET gratefully acknowledge support from the Leverhulme Trust during the course of this work.

\section{References}

Benson A. J., Lacey C. G., Baugh C. M., Cole S., \& Frenk C. S. 2002, MNRAS, 333,156

Bullock J. S., Kravtsov A. V., \& Weinberg D. H. 2000, ApJ, 539, 517

Dalal N., \& Kochanek C. S. 2002, ApJ, 572, 25

Dekel, A., \& Silk, J. 1986, ApJ, 303, 39

Ghigna S., et al. 2000, ApJ, 544, 616

Hayashi E., Navarro J. F., Taylor J. E., Stadel J., \& Quinn T. 2003, ApJ, 584, 541

Kauffmann G., White S. D. M., \& Guiderdoni B. 1993, MNRAS, 264, 201

Klypin A., Gottlöber S., Kravtsov A. V., \& Khokhlov A. M. 1999, ApJ, 516, 530

Klypin A., Zhao H., \& Somerville R. S. 2002, ApJ, 573, 597

Metcalf R. B. 2002, ApJ, 580, 696

Metcalf R. B., Moustakas L. A., Bunker A. J., Parry I. R. 2003, astro-ph/0309738

Moore B. 2000, astro-ph/0009247

Moore B., Governato F., Quinn T., Stadel J., \& Lake G. 1998, ApJ, 499, L5

Moore B., Ghigna S., Governato F., Lake G., Quinn T., Stadel J., \& Tozzi P. 1999, ApJ, 524, L19

Stoehr F., White S. D. M., Tormen G., \& Springel V. 2002, MNRAS, 335, L84

Taylor J. E. 2001, Ph.D. thesis, University of Victoria

Taylor J. E., \& Babul A. 2001, ApJ, 559, 716

Taylor J. E., \& Babul A. 2003, MNRAS, submitted (astro-ph/0301612)

White S. D. M. 1976, MNRAS, 177, 717

White S. D. M. 2000, ITP conference presentation, (online.itp.ucsb.edu/online/galaxy_c00/white)

White S. D. M. \& Rees, M. J. 1978, MNRAS, 183, 341 